\title{
Research on Handling Stability of Steering-by-wire System
}

\author{
Ying Yuan ${ }^{l, *}$, Guangxing Tan ${ }^{1}$ \\ ${ }^{1}$ College of Electrical and Information Engineering, Guangxi University of science and technology, Liuzhou 545006 China
}

\begin{abstract}
The main function of steer-by-wire (SBW) system are improving steering characteristics, security and stability of the vehicle. In this paper, the variable steering ratio of SBW system is analyzed, and the method of steering ratio based on fuzzy control and neural network are researched. In order to solve the actual working condition, the wheel angle may not reach the expected value, this paper establishes a twodegree-of-freedom (2-DOF) vehicle model, and a Matlab/Simulink simulation model, in which a control strategy based on PID controller is put forward to control the front wheel steering angle. Simulation results show that proposed control strategy based on fuzzy neural network can effectively reduce lateral deviation and improve the handling stability and comfortability of the vehicle.
\end{abstract}

\section{Introduction}

The steering system is the link between driver and vehicle, which plays an important role in the automobile active safety technology[1]. SBW system is a new technology of steering system, which improves the handling stability of the vehicle and reduces the burden on the driver. When driving, we mainly consider improving the handling stability of the vehicle[2]. The maneuverability means that the car can be driven in accordance with the driver's steering intention and not deviate from the intended trajectory. Stability refers to the ability of the car to maintain stable driving when disturbed by the outside world[3]. The centroid side-slip angle reflects the trajectory retention of vehicle, and the yaw rate is focused on the oversteer or understeer[4]. This paper first design the ideal steering ratio to guarantee yaw rate gain is a constant. And then the steering ratio based on fuzzy control and fuzzy neural network were discussed. Next, 2-DOF vehicle based on PID controller model is established, Finally, the vehicle output parameters are analyzed and compared to ensure the stability of the yaw rate and centroid side-slip angle.

\section{2-DOF vehicle dynamics model}

2-DOF vehicle handling stability model includes lateral motion, yaw motion and roll motion. Use the front wheel angle as input to observe the automobile steering movement. In the state of automobile movement, the output observation values are yaw rate $\gamma$, lateral acceleration $a_{r}$, and centroid side-slip angle $\beta$. Ignores the air resistance and tire's non-linear, the car model is established[5]. According to the lateral force balance and the balance of the moment around the centre of gravity:

$$
\left\{\begin{array}{l}
m V(\dot{\beta}+r)=-\left(P_{Y 1}+P_{Y 2}\right) \\
I \dot{r}=b P_{Y 2}-\alpha P_{Y 1}
\end{array}\right.
$$

Along with:

$$
\left\{\begin{array}{l}
P_{Y 1}=K_{1} \delta_{1}=K_{1}(\beta+\alpha r / V-\delta) \\
P_{Y 2}=K_{2} \delta_{2}=K_{2}(\beta-b r / V)
\end{array}\right.
$$

In the formula, we can simplify it as follow:

$$
\left\{\begin{array}{l}
m V(\dot{\beta}+r)=-\left(K_{1}+K_{2}\right) \beta+\left(b K_{2}-\alpha K_{1}\right) r / V+K_{1} \delta \\
I \dot{r}=\left(b K_{2}-\alpha K_{1}\right) \beta-\left(\alpha^{2} K_{1}+b^{2} K_{2}\right) r / V+\alpha K_{1} \delta \\
a_{r}=V(\beta+\dot{\gamma})
\end{array}\right.
$$

Above equation is subjected to a Laplace transform, that is, $r \rightarrow r(s), \beta \rightarrow \beta(s), \dot{r} \rightarrow \operatorname{sr}(s), \dot{\beta} \rightarrow s \beta(s)$, and make initial condition is 0 , then the transfer function is:

$\left\{\begin{array}{l}r / \delta(s)=\left(m V \alpha K_{1} s+l K_{1} K_{2}\right) /\{\Omega+\Phi s+\Psi\} \\ \beta / \delta(s)=\left(I K_{1} s+b l K_{1} K_{2} / V-m V \alpha K_{1}\right) /\{\Omega+\Phi s+\Psi\}\end{array}\right.$

Among them:

$$
\left\{\begin{array}{l}
\Omega=m V I s^{2} \\
\Phi=\left[I\left(K_{1}+K_{2}\right)+m\left(\alpha^{2} K_{1}+b^{2} K_{2}\right)\right] \\
\Psi=K_{1} K_{2} l^{2}\left(1+K V^{2}\right) / V
\end{array}\right.
$$

The $s$ is the Laplace variable in the formula, when written as standard transfer function form:

$$
\left\{\begin{array}{l}
r / \delta(s)=G_{r}\left(\tau_{1} s+1\right) /\left(T_{2} s^{2}+T_{1} s+1\right) \\
\beta / \delta(s)=G_{\beta}\left(\tau_{1}^{\prime} s+1\right) /\left(T_{2} s^{2}+T_{1} s+1\right)
\end{array}\right.
$$

Among them, $G_{r}$ and $G_{\beta}$ are steady-state gain:

$$
\left\{\begin{array}{l}
G_{r}=r /\left.\delta(s)\right|_{\delta=0}=V / l\left(1+K V^{2}\right) \\
G_{\beta}=\beta /\left.\delta(s)\right|_{\delta=0}=\left(b-V / C^{2}\right) / l\left(1+K V^{2}\right)
\end{array}\right.
$$

Among them:

$$
\left\{\begin{array}{l}
K=K_{1} / m b-K_{2} / m \alpha \\
T_{1}=V\left(b+\eta \alpha m b / K_{1} l+\alpha+\eta b m \alpha / K_{2} l\right) / l\left(1+K V^{2}\right) \\
T_{2}=I V^{2} K_{1} K_{2} l^{2} / m^{3} \alpha^{2} b^{2}\left(1+K V^{2}\right) \\
\tau_{1}=V K_{2} l / m \alpha \\
\tau_{1}^{\prime}=\eta /\left(K_{2} l / m \alpha V-V / b\right)
\end{array}\right.
$$

\footnotetext{
*Corresponding author: yrlsx99@126.com
} 
Table 1. Vehicle system parameter.

\begin{tabular}{|c|c|c|}
\hline Parameters/Unit & The physical meaning of parameters & Value \\
\hline $\mathrm{M} /(\mathrm{Kg})$ & Vehicle weight & 1359.8 \\
\hline$\alpha /(\mathrm{m})$ & Distance from mass center to front axle 1.06282 \\
\hline $\mathrm{l} /(\mathrm{m})$ & Wheelbase & 2.548 \\
\hline $\mathrm{K} 1 /(\mathrm{N} / \mathrm{rad})$ & Front tire cornering stiffness & 52480 \\
\hline $\mathrm{I} /(\mathrm{Kg} \cdot \mathrm{m} 2)$ & Moment of inertia & 1992.54 \\
\hline $\mathrm{b} /(\mathrm{m})$ & Distance from mass center to rear axle & 1.305 \\
\hline $\mathrm{K} 2 /(\mathrm{N} / \mathrm{rad})$ & Rear tire cornering stiffness & 88416 \\
\hline $\mathrm{K}$ & Stability factor & 0.0034 \\
\hline$\eta$ & Mass distribution coefficient & 0.9283 \\
\hline
\end{tabular}

\section{Design of ideal steering ratio}

In the research of steering characteristics, the steering ratio under steady-state steering gain is the most important. As the steering ratio of the SBW is variable, in order to reduce the burden of the driver, it can be designed as follows: The steering ratio is small when the car is driving at low speed, so the driver turning a small steering handle angle can have a greater steering wheel angle[6]. When the speed is high, the steering ratio is large to ensure that the steering stability in the process of steering. Thereby simplifying driver's steering operation and improving the active safety of automotive SBW[7].

The steering ratio of SBW is determined by the front wheel angle $\delta$ and the steering wheel angle $\delta_{h}$, which can be expressed as $i$ :

$$
\delta=\delta_{h} / i
$$

Two steering gains are defined in the vehicle dynamics. One of steering gain refers to the ratio of front wheel angle $\delta$ and yaw rate $\omega_{r}$, represented by $G_{\delta}^{\omega_{r}}$. And the steady-state conditions are as follows:

$$
G_{\delta}^{\omega_{r}}=\omega_{r} / \delta
$$

Another steering gain $G_{\delta_{h}}^{\omega_{r}}$ refers to steering wheel angle $\delta_{h}$ and yaw rate $\omega_{r}$, under the steady-state conditions:

$$
G_{\delta_{h}}^{\omega_{r}}=\omega_{r} / \delta_{h}
$$

If the steering wheel angle is linearly related to the road travel angle, that is, $G_{\delta}^{\omega_{r}} G_{s}=K_{\mathrm{s}}$ is a constant, Where $G_{s}=1 / i$. Then the steering operation can be simplified and the driver's burden can be reduced. The ideal steering ratio is variable and yaw rate gain $K_{s}$ is constant. The steering characteristics of ideal steering ratio are easily to be mastered by the driver.

$$
i=G_{\delta}^{\omega_{r}} / K_{\mathrm{s}}=v / K_{\mathrm{s}} L\left(1+K v^{2}\right)
$$

In above formula: $K=m\left(a / k_{2}-b / k_{1}\right) / L^{2}, L=a+b$.

When the car is low speed or static steering, the larger static friction force makes the steering resistance torque become large, in order to make the steering "light", transmission ratio should be smaller and constant. Substituting the data of the Volvo 264 model[8], the maximum steering wheel angle of car is $420^{\circ}$, the maximum of front wheel angle is $45^{\circ}$. The transition speed $v_{0}=30 \mathrm{~km} / \mathrm{h}$ is selected from "Fig. 1", so the minimum value of steering ratio $i$ :

$$
i_{\text {min }}=\delta_{h \max } / \delta_{\max }=420 / 45 \approx 9.333
$$

Therefore, the ideal steering ratio based on the yaw rate gain $K_{s}$ constant should be:

$$
i=\left\{\begin{array}{ccc}
9.333 & (v<30 & \mathrm{km} / \mathrm{h}) \\
v / K_{\mathrm{s}} L\left(1+K v^{2}\right) & (v \geq 30 & \mathrm{km} / \mathrm{h})
\end{array}\right.
$$

In order to select a reasonable yaw rate gain $K_{s}$, the values of $K_{s}$ are $0.19,0.27,0.32,0.45$, and the following curves are obtained.

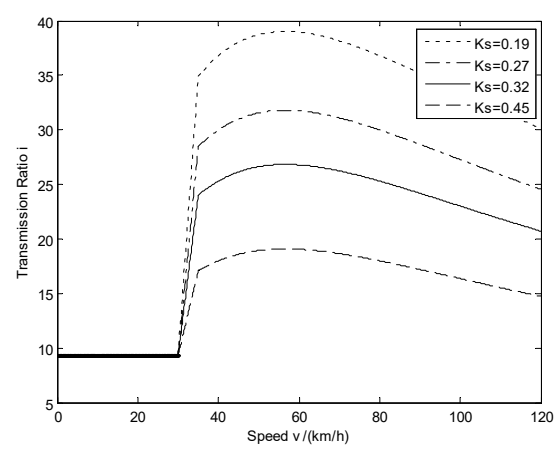

Fig. 1. Ideal steering ratio curves with different speed.

The figure shows that when the car driving slowly, it is possible to improve the steering sensitivity of SBW by using a constant and smaller steering ratio. In the high speed driving, the driver can avoid the fault operation. It is advantageous to improve the safety and stability of car.

\section{Design of fuzzy steering ratio}

Generally, the steering ratio should be kept between 15:1 and 20:1. From the above figure, the ideal steering ratio does not meet the actual requirements of the steering system[9]. Considering the influence of vehicle speed and steering wheel angle, this paper use fuzzy control algorithm to establish a more reasonable steering ratio of SBW system. Based on fuzzy control toolbox of Matlab, the selected fuzzy subset language is $\{\mathrm{NB}, \mathrm{NM}, \mathrm{NS}, 0$, PS, PM , PB $\}$. Fuzzy rules are designed as follows:

$v=\{N B, N M, N S, 0, P S, P M, P B\}$, the language values are $\{0,20,40,60,80,100,120\}$.

$\delta_{h}=\{N B, N M, N S, 0, P S, P M, P B\}$, the language values are $\{0,70,140,210,280,350,420\}$.

$i=\{N B, N M, N S, 0, P S, P M, P B\}$, the language values are $\{3,6,9,12,15,18,21\}$.

The fuzzy controller is double input and single output, and membership function graphs are Gaussian curves. Based on the driver's driving behavior and expert knowledge, the fuzzy steering ratio control table is established as follows.

Table 2. Rule of steering ratio fuzzy control.

\begin{tabular}{|c|c|c|c|c|c|c|c|}
\hline \multirow{2}{*}{$\mathbf{v}$} & \multicolumn{7}{|c|}{$\boldsymbol{\delta}_{\mathbf{h}}$} \\
\cline { 2 - 8 } & $\boldsymbol{N} \boldsymbol{B}$ & $\boldsymbol{N} \boldsymbol{M}$ & $\boldsymbol{N} \boldsymbol{S}$ & $\boldsymbol{Z}$ & $\boldsymbol{P S}$ & $\boldsymbol{P} \boldsymbol{M}$ & $\boldsymbol{P} \boldsymbol{B}$ \\
\hline $\boldsymbol{N} \boldsymbol{B}$ & NB & NB & NB & NB & NB & NB & NB \\
\hline $\boldsymbol{N} \boldsymbol{M}$ & NB & NB & NB & NB & NB & NB & NB \\
\hline $\boldsymbol{N} \boldsymbol{S}$ & NM & NS & NS & Z & NS & NS & NM \\
\hline $\boldsymbol{Z}$ & Z & PS & PS & PM & PS & PS & Z \\
\hline $\boldsymbol{P S}$ & PS & PM & PM & P & PM & PM & PS \\
\hline $\boldsymbol{P M}$ & PM & PM & PM & PB & PM & PM & PM \\
\hline $\boldsymbol{P B}$ & PB & PB & PB & PB & PB & PB & PB \\
\hline
\end{tabular}

According to the fuzzy rule table, the steering ratio curve surface diagram as follows: 


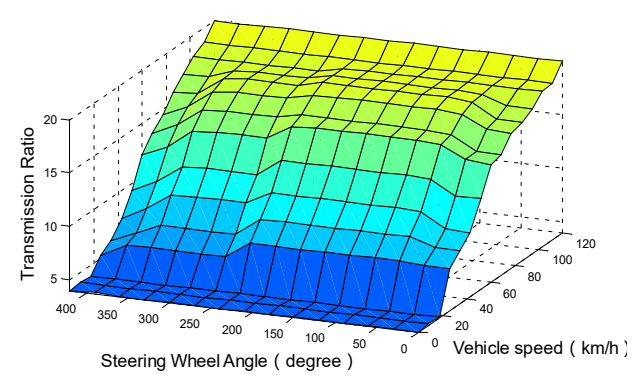

Fig. 2. Relationship between $i, v$ and $\delta_{h}$.

It can be seen from the figure, as the speed increases, the steering ratio increases gradually. So as to effectively improve the vehicle handling stability and reduce the incidence of traffic accidents. But the fuzzy controller output curve is not smooth enough.

\section{Fuzzy neural network makes better steering ratio}

Because of fuzzy control relies too much on expertise, so used the fuzzy neural network algorithm to improve the design. In order to use fuzzy neural network to adjust membership rules and functions of fuzzy control, the steering ratio of two inputs and an output fuzzy neural network model is established[10]. Based on the fuzzy control system model, the fuzzy network structure is based on the extended radial basis function (RBF) neural network, which functions is a TS fuzzy inference system.

There are 5 layers of network structure:

The first layer: input layer, the number of nodes is 2 , that is, steering wheel angle and speed.

$$
f(X)=\left[x_{1}, x_{2}\right]
$$

The second layer: fuzzy layer, 14 nodes, the role is to complete the input of fuzzy.

$$
\mu_{i}^{j}\left(x_{i}\right)=\exp \left[-\left(x_{i}-c i_{j}\right)^{2} / \sigma_{j}^{2}\right]
$$

The third layer: fuzzy inference layer, 49 nodes, the role is to achieve conditional fuzzy set operations.

$$
\varphi_{j}=\mu_{1}^{i_{1}} \mu_{2}^{i_{2}}, i_{1}=1,2, \ldots m_{1}, i_{1}=1,2, \ldots m_{2}, m=m_{1} m_{2}
$$

Fourth layer: normalization calculation.

$$
a_{j}=\varphi_{j} / \sum_{k=1}^{\varphi_{j}} \varphi_{k}
$$

The fifth layer: output layer, that is transmission ratio.

$$
y(X)=\sum_{j=1}^{m} a_{j} \omega_{j}, \omega_{j}=p_{j 0}+p_{j 1} x_{1}+p_{j 2} x_{2}
$$

Given a typical speed and a different steering wheel angle as input, the fuzzy control will produce the corresponding steering ratio. After repeated operation, save vehicle data in various circumstances and establish a database. And then some training data as follows.

Table 3. A part of the training data.

\begin{tabular}{|c|c|c|c|c|c|c|c|c|}
\hline $\mathbf{V}$ & $\boldsymbol{\delta}_{\mathbf{h}}$ & $\mathbf{i}$ & $\mathbf{V}$ & $\boldsymbol{\delta}_{\mathbf{h}}$ & $\mathbf{i}$ & $\mathbf{V}$ & $\boldsymbol{\delta}_{\mathbf{h}}$ & $\mathbf{i}$ \\
\hline 30 & -210 & 8.10 & 70 & -210 & 12.32 & 110 & -210 & 15.48 \\
\hline 30 & -140 & 8.78 & 70 & -140 & 13.76 & 110 & -140 & 15.98 \\
\hline 30 & -70 & 9.20 & 70 & -70 & 14.14 & 110 & -70 & 16.68 \\
\hline 30 & 0 & 9.88 & 70 & 0 & 15.21 & 110 & 0 & 17.32 \\
\hline 30 & 70 & 9.23 & 70 & 70 & 13.98 & 110 & 70 & 16.57 \\
\hline 30 & 140 & 8.84 & 70 & 140 & 13.10 & 110 & 140 & 15.80 \\
\hline 30 & 210 & 8.12 & 70 & 210 & 12.43 & 110 & 210 & 15.41 \\
\hline
\end{tabular}

The membership functions of the input variables are optimized as follows: from the figure we can see that the central value and width of the membership functions have a certain degree of adjustment.
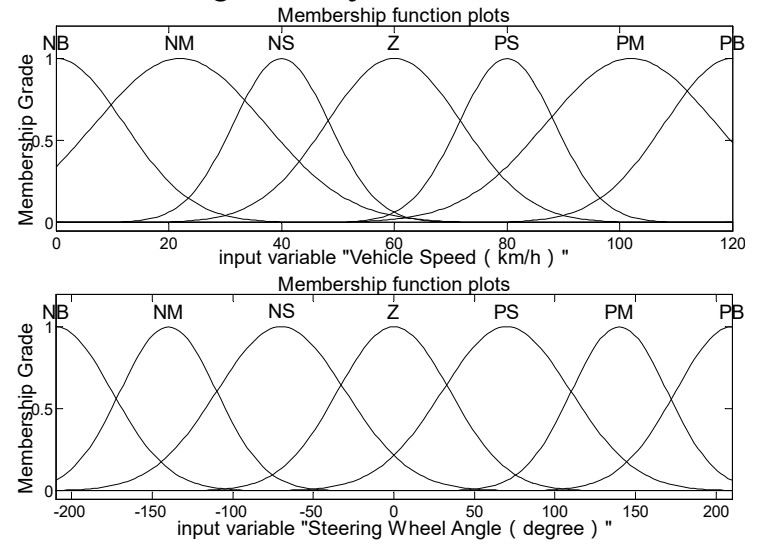

Fig. 3. Membership function of input variable after optimization.

\section{Design of PID controller for two degree-of-freedom vehicle model}

The driver turns the steering wheel according to the speed of the vehicle under different conditions, and the corresponding steering wheel angle expectation value is obtained by designing the variable transmission ratio model. Therefore, in this paper, the yaw rate and centroid side angle of the steering vehicle are taken as the control target, and the control stability of the vehicle is analyzed by PID control. The system input is front wheel angle determined by fuzzy neural network ratio of SBW system. Using the steering response of SBW system to better track the vehicle model and the smaller error evaluation index set controller: The conventional PID controller input is the difference $e(t)$ between the expected value $r(t)$ and the actual output value $y(t)$. Used proportional operation, integral operation and derivative operation for the $e(t)$, and a proper control quantity $u(t)$ is obtained by summation.

The PID control transfer function is:

$$
G(s)=K_{p}+K_{i} / s+K_{d} s
$$

The mathematical equation of the PID controller is:

$$
y(X)=\sum_{j=1}^{m} a_{j} \omega_{j}, \omega_{j}=p_{j 0}+p_{j 1} x_{1}+p_{j 2} x_{2}
$$

In which, $e(t)=u(t)-y(t), K_{P}$ is the coefficient of proportionality, $T_{i}=K_{P} / K_{i}$ is integral time constant, $T_{d}=K_{d} / K_{P}$ is differential time constant. Based on Matlab/Simulink software, build a simulation model as follow:

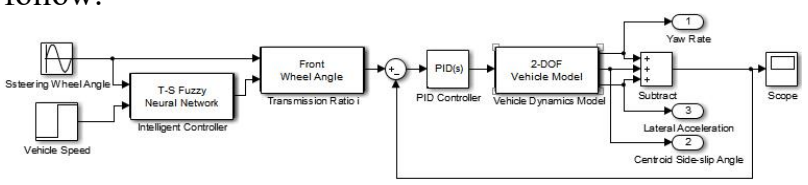

Fig. 4. Simulation model of PID control for SBW system based on fuzzy neural network ratio.

First set the initial value of the three parameters of the PID, generally the value of $K_{P}$ is given first, and the 
$K_{i}$ and $K_{d}$ are set to 0 , which can avoid overshoot and improve the response speed. In this paper, the system input is a single lane change. $K_{d}$ can improve the dynamic characteristics of the system, restrain the controlled variable, shorten the adjustment time and reduce the steady-state error. Through several simulation comparisons, the output parameters of the automotive SBW system are simulated as follows:

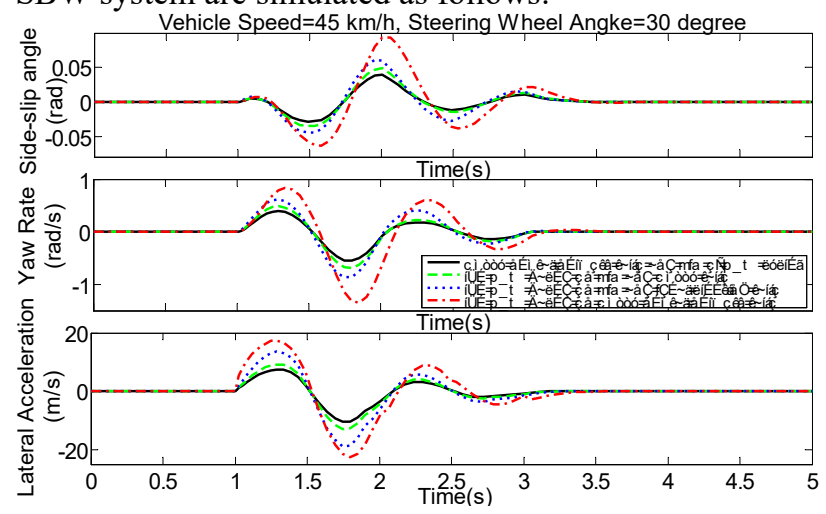

Fig. 5. Curve of parameter change in vehicle steering process.

Table 4. Simulation result comparison analysis.

\begin{tabular}{|c|c|c|c|c|c|}
\hline \multicolumn{2}{|c|}{$\begin{array}{l}\text { The vehicle output } \\
\text { parameters }\end{array}$} & \multirow{2}{*}{\begin{tabular}{|c|}
$\begin{array}{c}\text { Fuzzy neural } \\
\text { network ratio } \\
\text { and PID of } \\
\text { SBW system }\end{array}$ \\
0.0392 \\
\end{tabular}} & \multirow{2}{*}{$\begin{array}{c}\begin{array}{c}\text { Fuzzy ratio } \\
\text { and PID of } \\
\text { SBW system }\end{array} \\
0.0487\end{array}$} & \multirow{2}{*}{\begin{tabular}{|c|}
$\begin{array}{c}\text { Ideal ratio } \\
\text { and PID of } \\
\text { SBW system }\end{array}$ \\
0.0579
\end{tabular}} & \multirow{2}{*}{\begin{tabular}{|c|}
$\begin{array}{c}\text { Fuzzy neural } \\
\text { network ratio } \\
\text { of SBW } \\
\text { system }\end{array}$ \\
0.0933 \\
\end{tabular}} \\
\hline \multirow{2}{*}{$\begin{array}{l}\text { Side-slip } \\
\text { angle }\end{array}$} & $\begin{array}{c}\text { Maximum } \\
(\mathrm{rad} / \mathrm{s})\end{array}$ & & & & \\
\hline & $\begin{array}{l}\text { Settling } \\
\text { time(s) }\end{array}$ & 3.3639 & 3.4139 & 3.3639 & 3.7639 \\
\hline \multirow{2}{*}{ Yaw rate } & $\begin{array}{c}\text { Minimum } \\
(\mathrm{rad} / \mathrm{s})\end{array}$ & -0.5538 & -0.6884 & -0.8589 & -1.3468 \\
\hline & $\begin{array}{l}\text { Settling } \\
\text { time(s) }\end{array}$ & 3.2139 & 3.3639 & 3.2639 & 3.9639 \\
\hline \multirow{2}{*}{$\begin{array}{l}\text { Lateral } \\
\text { acceleration }\end{array}$} & $\begin{array}{c}\text { Minimum } \\
(\mathrm{rad} / \mathrm{s})\end{array}$ & -10.4499 & -12.8488 & -18.7831 & -22.6140 \\
\hline & $\begin{array}{l}\text { Settling } \\
\text { time(s) }\end{array}$ & 3.6639 & 3.7639 & 3.7639 & 3.9639 \\
\hline
\end{tabular}

The graphs show the different algorithms of steering ratio on SBW system PID control model. Since the yaw rate and lateral acceleration mainly focus on describing vehicle stability, the centroid side-slip angle reflects vehicle trajectory maintenance. Therefore, the vehicle stabilization time is shorter, indicating that the vehicle has good handling stability. From graphs data show that the output parameters of SBW system based on PID and fuzzy neural network control are lower than others. Without PID controller, the SBW system has the longest stability time, and the curve of lateral acceleration is not smooth. The results proved that the PID control model of automobile SBW system based on fuzzy neural network steering ratio can improved the steering characteristics and the handling stability, reduce the burden on driver.

\section{Conclusion}

As can be seen from this paper, the ideal transmission ratio is difficult to meet the actual requirements. To solve this problem, this paper establishes a two-degreeof-freedom vehicle model, and the fuzzy neural network control algorithm is used to optimize the steering ratio. The system can improve steering sensitivity of vehicle at low speed, and in the high speed driving, the driver can be prevented from fault operation, thereby improve the handling stability. The simulation results show that use the PID controller to adjust SBW system of fuzzy neural network steering ratio, the vehicle output parameters can be stabilized in a short time, so as to meet the requirements of vehicle steering sensitivity at different speeds. This system reduces burden of the driver, improve the vehicle handling stability and driving safety.

\section{Acknowledgments}

Project supported by the Major Program of the National Natural Science Foundation of China (61563005) and Innovation Project of GuangXi University of Science and Technology Graduate Education (GKYC201709).

\section{References}

1. C. Wang, T. Cui, W. Zhao, et al. Active front wheel steering control based on ideal transmission ratio. Transactions of the Chinese Society of Agricultural Engineering, 31(4):85-90(6),(2014)

2. Q. Zhang. Research on Modeling and Control Strategy of Electric Forklift Steer-by-wire System. HeFei University of Technology, (2015)

3. S. Tang. Research on Automobile Steer by Wire System Based on Fuzzy Adaptive PID Control. Shandong University of Technology, (2011)

4. Y. Liu. Research on Modeling and Active Steering Control Algorithm for Electric Forklift Steer-byWire System. MECS, 8(11):70-79, (2016)

5. L. Yu. On Lateral Stability Analysis of Steering-byWire System Based on Fuzzy PID Control. Journal of Shandong Jiaotong University, (2014)

6. G. Wang. Study on Variable Steering Ratio and Active Steering Control Strategy of Steer-by-wire System. Liaoning University of Technology, (2015)

7. Q. Cao, G. Tan. Research on front wheel of steer by wire system based on fuzzy Immune PID. Guangxi University of Science and Technology, (2014).

8. J. Tian. Research on Dynamic Analysis and Control Method of Steer-by-wire System for Vehicle. Jiangsu University, (2011)

9. K. Zhang, S. Cui, J. Wang. Path Tracking Control of Intelligent Vehicle Based on Fuzzy Neural Network. Automotive Engineering, 37(1):38-42 and 77, (2015)

10. N. Wang, J. Shi, G. Li. Study on Active Steering Control Strategy for Steer-by-Wire System. Automobile Applied Technology, 1:72-73, (2016). 